\title{
Doença Hepática Gordurosa Não Alcoólica e Síndrome Metabólica
}

\author{
Non-Alcoholic Fatty Liver Disease and Metabolic Syndrome \\ Enfermedad del Hígado Graso no Alcohólico y Síndrome Metabólico
}

\begin{abstract}
Victor Fernando Costa Macedo Noronha1*, Sônia Oliveira Lima ${ }^{1}$, Allan Victor Hora Mota ${ }^{1}$, Yasmin Hora Góis Gonzaga ${ }^{1}$, Nathalia Nascimento Santana ${ }^{1}$, Jéssica Teles Santana ${ }^{1}$, Raphaella Maria Oliveira Pereira Gomes ${ }^{1}$, Carla Pérez Machado², Anny Carolyne Oliveira Lima Santos ${ }^{1}$, Josilda Ferreira Cruz ${ }^{1}$.
\end{abstract}

\section{RESUMO}

Objetivo: Avaliar a associação entre doença hepática gordurosa não alcoólica (DHGNA) e síndrome metabólica (SM). Métodos: Estudo clínico, prospectivo, tipo survey, com dados coletados em um centro de referência em ultrassonografia em Aracaju-SE. O processo de coleta de dados foi dividido em três etapas. Os dados obtidos foram analisados estatisticamente pelo software $R$, versão 3.6.1. Resultados: Foram analisados 527 pacientes, de ambos os sexos, com idade entre 14 e 89 anos. Na amostra, $29,1 \%$ tiveram algum grau de esteatose hepática não alcoólica e 70,9\% não apresentavam infiltração gordurosa no fígado. A maioria dos pacientes era do sexo feminino $(n=356 ; 67,6 \%)$ e não era portadora da $S M(n=410 ; 79,5 \%)$. A ausência da SM mostrou menor tendência de o paciente apresentar algum grau de gordura hepática. Houve associação estatisticamente significativa entre circunferência abdominal (CC), índice de massa corpórea (IMC) e peso corporal em relação aos graus da esteatose hepática. Conclusão: As medidas antropométricas de IMC, CC e peso corporal, assim como a presença de SM se apresentaram como fatores de gravidade para DHGNA.

Palavras-chave: Doença hepática gordurosa não alcoólica, Síndrome metabólica, Ultrassonografia, Circunferência da cintura, Índice de massa corporal.

\begin{abstract}
Objective: To evaluate the association between Non-Alcoholic Fatty Liver Disease (NAFLD) and Metabolic Syndrome (MS). Methods: linical and prospective study, survey type, with data collected in an ultrasound reference center in Aracaju-SE. The data collection process was divided in three stages. The obtained data were analyzed statistically by software R, version 3.6.1. Results: A total of 527 patients, of both sexes, aged between 14 and 89 years old, were analyzed. In the sample, $29.1 \%$ had some degree of non-alcoholic liver steatosis and $70.9 \%$ did not have fatty infiltration in the liver. Most patients were female $(n=356 ; 67.6 \%)$ and did not have MS $(n=410 ; 79.5 \%)$. The absence of MS showed a lower tendency for the patient to present some degree of hepatic fat. There was a statistically significant association between Waist Circumference (WC), Body Mass Index (BMI) and body weight in relation to the degrees of hepatic steatosis. Conclusion: Anthropometric measurements of BMI, WC and body weight, as well as the presence of MS, were presented as factors of severity for NAFLD.
\end{abstract}

Keywords: Non-alcoholic fatty liver disease, Metabolic syndrome, Ultrasonography, Waist circumference, Body mass index.

\footnotetext{
1Universidade Tiradentes (UNIT). Aracaju - SE. *E-mail: victornoronha96@gmail.com

${ }^{2}$ Hospital Beneficência Portuguesa de São Paulo. São Paulo - SP.
} 


\section{RESUMEN}

Objetivo: Evaluar la asociación entre la Enfermedad del Hígado Graso No Alcohólico (EHGNA) y el síndrome metabólico (SM). Métodos: Estudio clínico prospectivo, tipo de encuesta, con datos recopilados en un centro de referencia de ultrasonido en Aracaju-SE. El proceso de recopilación de datos se dividió en tres etapas. Los datos obtenidos fueron analizados estadísticamente por el software R, versión 3.6.1. Resultados: Se analizaron 527 pacientes, de ambos los sexos, con edades comprendidas entre 14 y 89 años. En la muestra, el $29.1 \%$ tenía algún grado de esteatosis hepática no alcohólica y el $70.9 \%$ no tenía infiltración grasa en el hígado. La mayoría de los pacientes eran mujeres ( $n=356 ; 67,6 \%)$ y no tenían SM ( $=410 ; 79,5 \%$ ). La ausencia del SM mostró una menor tendencia para que el paciente presente algún grado de grasa hepática. Hubo una asociación estadísticamente significativa entre la circunferencia de la cintura (CC), el índice de masa corporal (IMC) y el peso corporal en relación con los grados de esteatosis hepática. Conclusión: Las medidas antropométricas de IMC, WC y peso corporal, así como la presencia de EM, se presentaron como factores de gravedad para la EHGNA.

Palabras clave: Enfermedad del hígado graso no alcohólico, Síndrome metabólico, Ultrasonografía, Circunferencia de la cintura, Índice de masa corporal.

\section{INTRODUÇÃO}

O fígado é um centro de numerosos processos fisiológicos, como metabolismo dos macronutrientes, a regulação do volume sanguíneo, o suporte do sistema imunológico, o controle endócrino das vias de sinalização do crescimento, a homeostase dos lipídios e colesterol e a quebra dos compostos xenobióticos (TREFTS E, et al. 2017).

A doença hepática gordurosa não alcoólica (DHGNA) abrange um amplo espectro de doença hepática, desde esteatose hepática com acúmulo de gordura nos hepatócitos até esteato-hepatite não alcoólica (EHNA), com inflamação e lesão adicional dos hepatócitos à cirrose hepática, insuficiência hepática e carcinoma hepatocelular (CHC) (KIRKEGAARD-KLITBO DM, et al., 2020). Associa-se a distúrbios metabólicos, como diabetes, obesidade, hiperlipidemia e hipertensão arterial sistêmica (HAS) (YE Q, et al.).

Devido ao rápido aumento das taxas de obesidade e diabetes mellitus tipo II (DM2), a DHGNA é considerada a forma mais comum de doença hepática crônica nos países desenvolvidos, ajudando a sobrecarregar os sistemas de saúde em todo o mundo (HUANG T, et al., 2019). A DHGNA é definida como o desenvolvimento de infiltração gordurosa em maior que $5 \%$ dos hepatócitos identificados histologicamente ou radiologicamente, na ausência de causas secundárias, como hepatite viral, álcool ou doenças hereditárias do fígado (HUANG T, et al., 2019). A esteatose hepática não alcoólica é descrita como a presença de $\geqq 5 \%$ de esteatose hepática sem evidência de lesão hepatocelular. A EHNA, é classificada pela presença de $\geqq 5 \%$ de esteatose hepática e inflamação lobular com lesão de hepatócitos, com ou sem fibrose (REDDY YK, et al., 2019).

A base fisiopatológica da DHGNA tem grande influência ambiental atrelada a suscetibilidade genética (FEDERICO A, et al., 2020). Percebe-se que a síndrome metabólica (SM) e a resistência insulínica (RI) tem papel fundamental na patogênese desta afecção, que envolve acúmulo de triglicerídeos (TG) intra-hepáticos e inflamação (TROVATO FM, et al., 2019). O acúmulo de ácidos graxos resulta em lipotoxicidade e resistência à insulina e, juntamente com endotoxinas derivadas do intestino, desencadeiam uma cascata de resposta inflamatória ao liberar citocinas pró-inflamatórias no fígado (TAN HL, et al., 2020).

Estima-se que fatores genéticos e epigenéticos contribuam com cerca de 20 a $70 \%$ na patogênese da DHGNA por meio da herdabilidade. Vários estudos genômicos descobriram a presença de numerosos polimorfismos de nucleotídeo único, variações na sequência de DNA que afetam apenas uma base da sequência genômica, de genes como o domínio fosfolipase do tipo patatina contendo proteína-3 (PNPLA3) para relacionar-se independentemente com a gravidade da doença, progressão e risco de CHC (HUANG T, et al., 2019). 
Por conta da alta prevalência da doença e aos riscos multissistêmicos, o diagnóstico da DHGNA requer uma abordagem bastante cautelosa. Consequentemente, as diretrizes da Associações Européias para o Estudo do Fígado, Diabetes e Obesidade (EASL/EASD/EASO) concentram-se em modalidades de diagnóstico simples e econômicas, além de estratificar o risco de pacientes que necessitam de monitoramento clínico a longo prazo ou intervenção terapêutica, permanecendo o menos invasivo possível e minimizando a necessidade de uma biópsia hepática (PAPATHEODORIDI M e CHOLONGITAS E, 2018).

A ultrassonografia (USG) é considerada o estudo de imagem de primeira linha. $\mathrm{Na}$ imagem ultrassonográfica, a alteração esteatótica do fígado pode ser visualizada como hiperecogenicidade do órgão devido a vacúolos de lipídios, obscurecimento das margens vasculares e heterogeneidade do parênquima (SWEET PH, et al., 2017).

A sensibilidade e especificidade do ultrassom são de aproximadamente $85 \%$ e $90 \%$, respectivamente (HUANG T, et al., 2019). A USG tem a vantagem significativa de ser não invasiva, livre de radiação, facilmente disponível e pouco onerosa (JENNISON E, et al., 2019).

A tomografia computadorizada (TC) tem alta especificidade para identificar outras causas de doença hepática, mas é mais cara que a ecografia e o risco potencial de radiação ionizante a torna inadequada para o monitoramento longitudinal de pacientes com DHGNA (SWEET PH, et al., 2017; JENNISON E, et al., 2019).

A ressonância magnética $(\mathrm{RM})$ também pode ser útil na detecção de esteatose hepática (JENNISON E, et al., 2019). A biópsia hepática ainda permanece o padrão-ouro em pacientes com DHGNA na definição de esteato-hepatite e avaliação da fibrose, embora sejam bem conhecidas desvantagens que limitam sua utilização, como custo, erro de amostragem, variabilidade inter/intra-observador e risco relacionado ao procedimento (PAPATHEODORIDI M e CHOLONGITAS E, 2018).

Os princípios gerais para o gerenciamento da DHGNA envolvem a identificação precoce das pessoas em risco, modificação dos fatores de risco metabólicos, monitoramento da progressão para doença hepática avançada e prevenção de lesões hepáticas concomitantes por outros fatores, como o álcool (HUANG T, et al., 2019). A gordura hepática está intimamente relacionada ao risco cardiometabólico e, para diminuir a incidência lipídica intra-hepática e melhorar a DHGNA, são recomendadas intervenções no estilo de vida envolvendo dieta e atividade física (TROVATO FM, et al., 2019).

O grau de fibrose em pacientes com DHGNA é a única característica histológica do prognóstico a longo prazo na doença, além de estratificar o risco da mortalidade cardiovascular e por outras as causas. Logo, pacientes com risco de progressão para EHNA e fibrose hepática devem ser identificados para impedir piores prognósticos (KIRKEGAARD-KLITBO DM, et al., 2020; REDDY YK, et al., 2019).

A SM é uma condição sistêmica gerada por um amplo espectro de características clínicas como obesidade central, HAS e comprometimento da glicose e homeostase lipídica. (BATTAGLIA S, et al., 2020). Os mecanismos patogênicos da SM são complexos e ainda precisam ser totalmente elucidados, agrupando fatores que promovem o desenvolvimento de vários distúrbios, incluindo doenças cardiovasculares, DM2, câncer, DHGNA, doença renal crônica, hiperuricemia e gota (WANG L, et al., 2020).

A adiposidade visceral tem demonstrado ser um "gatilho" primário para a maioria das vias envolvidas, enfatizando a importância de uma alta ingestão calórica como principal fator causador. De todos os mecanismos propostos, RI, ativação neuro-hormonal e inflamação crônica parecem ser os principais elementos no início, progressão e transição da SM para doença cardiovascular aterosclerótica (ROCHLANI Y, et al., 2017).

Em 2006, a Federação Internacional de Diabetes (IDF) atualizou os critérios para diagnóstico da SM e levou em consideração sexo, etnia, glicose plasmática em jejum concentração de lipoproteína de densidade alta (HDL) , níveis de TG, pressão arterial sistólica (PAS) e pressão arterial diastólica (PAD) $\geq 85$ 
mmHg, circunferência da cintura (CC) e tratamento para diabetes, dislipidemia e hipertensão (INTERNATIONAL DIABETES FEDERATION, 2006).

Com o aumento da obesidade nos países ocidentais, a prevalência de DHGNA seguiu um crescimento semelhante. Como a esteatose hepática não alcoólica está intimamente associada à obesidade, RI, hipertensão arterial e dislipidemia, é considerada a manifestação hepática da SM (DIETRICH P e HELLERBRAND C, 2014).

A DHGNA e SM se sobrepõem em diversos aspectos, incluindo o espectro histopatológico de acometimentos hepático que elas predizem e do fato da SM ser um importante preditor de EHNA. A presença de uma dessas doenças pode diferenciar o motivo pelo qual alguns, mas não todos os indivíduos obesos e mesmo alguns não obesos, desenvolvem complicações metabólicas adicionais (YKI-JÄRVINEN H, 2014). Em um estudo a USG revelou infiltração gordurosa do fígado em aproximadamente $50 \%$ dos pacientes com hiperlipidemia e que em pacientes com DM2 a associação com DHGNA é bem reconhecida (DIETRICH P e HELLERBRAND C, 2014).

O objetivo do presente estudo é analisar a associação entre a esteatose hepática não alcoólica diagnosticada pela USG e a presença de SM pelos critérios do IDF, permitindo detectar os fatores da SM nos portadores de DHGNA e direcionar as intervenções terapêuticas para diminuir as repercussões prejudiciais a saúde do indivíduo.

\section{MÉTODOS}

Estudo clínico, prospectivo, tipo survey, com abordagem analítica quantitativa. Os dados foram coletados em um centro de referência de ultrassonografia no município de Aracaju - SE, Brasil, no período entre março/2019 a março/2020 após aprovação do Comitê de Ética em Pesquisa da Universidade Tiradentes através do cadastro $010513 \mathrm{R}$ e seguiu as diretrizes e normas para estudo com seres humanos. Os exames foram realizados por um único examinador, com o mesmo aparelho de USG e com boa resolução de imagem.

Os critérios de inclusão foram indivíduos de ambos os sexos de 14 a 89 anos de idade. Os critérios de exclusão foram: pacientes com consumo de álcool $\geq 140 \mathrm{~g} / \mathrm{semana}$ (sexo masculino) e $\geq 70 \mathrm{~g} /$ semana (sexo feminino), portadores de doenças hepáticas crônicas ou/e de tumor primário no fígado, portadores de deficiência cognitiva e em uso regular de drogas indutoras de esteatose hepática como amiodarona, tamoxifeno, corticoesteróides, diltiazem e antirretroviral

Foram analisadas as seguintes variáveis: nome, sexo, idade, circunferência da cintura, peso, IMC, níveis pressóricos ou uso de anti-hipertensivos, glicemia de jejum ou uso de hipoglicemiantes, triglicerídeos e HDL ou de drogas para dislipidemia em relação a variável esteatose hepática não alcoólica.O procedimento de coleta dividiu-se em três etapas: 01, 02 e 03.

$\mathrm{Na}$ etapa 01 foram seguidos oito passos: Assinatura do Termo de Consentimento Livre e Esclarecido; Preenchimento do questionário sociodemográfico contendo as variáveis: nome, idade, sexo, consumo de álcool, ausência de doenças hepáticas crônicas ou tumores primários no fígado e uso de medicamentos indutores de esteatose hepática e para tratamento de HAS e DM2; Cálculo do consumo etílico utilizando a fórmula: dose em $\mathrm{ml} \times$ grau $\times 0,8 / 100$, onde os graus ou teor alcoólico das bebidas são conhecidos, a saber os seguintes teores etílicos: cerveja 4 , vinho 12 , conhaque 40, rum 40, uísque 43, pinga 46 (MINCIS M e MINCIS R, 2011); Quantificação do peso corpóreo com a balança portátil marca TECHLINE e para isso o paciente permaneceu em pé, descalço no centro da balança e com o peso distribuído em ambos os pés; Aferição da altura com o estadiômetro portátil da marca FILIZOLA com a leitura realizada no milímetro (mm) mais próximo, tendo o paciente se posicionado descalço, verticalmente com os braços estendidos ao longo do corpo, ombros relaxados e com os calcanhares juntos e a cabeça posicionada; Cálculo do IMC pelo índice de Quetelet, pela razão entre o peso corpóreo em quilograma e altura em metro ao quadrado (WHO, 1995); Mensuração da CC com o paciente em pé, utilizando uma fita métrica inelástica a nível do ponto 
médio entre a última costela e a crista ilíaca (LOHMAN TG, et al., 1988); Aferição da PA com estetoscópio da marca Littmann do modelo Classic III e um esfigmomanômetro da marca Premium calibrado e dentro das recomendações do INMETRO, seguindo os critérios da Sociedade Brasileira De Cardiologia, et al. (2018).

$\mathrm{Na}$ etapa 02 foi realizado o exame de ultrassonografia abdominal modo $\mathrm{B}$, com transdutor convexo, dinâmico (com formação da imagem contínua e automática), de frequência de $3,75 \mathrm{MHz}$. Os pacientes se encontravam com preparo adequado, ou seja, jejum de no mínimo 6 horas e uso de antiflatulento. A esteatose hepática não alcoólica foi classificada em graus de acordo com Saadeh SY, et al. (2002): Grau 0: Ecogenicidade normal; Grau 1: Esteatose leve, com visualização de ecos finos do parênquima hepático, visualização normal do diafragma e dos vasos intra-hepáticos; Grau 2: Esteatose moderada, com aumento difuso nos ecos finos, visualização prejudicada dos vasos intra-hepáticos e do diafragma; Grau 3: Esteatose severa, com um aumento importante dos ecos finos, visualização prejudicada ou ausente dos vasos intrahepáticos.

$\mathrm{Na}$ etapa 03, após jejum de 12-14 horas e de acordo com as normas internas do laboratório, foram realizados exames bioquímicos de dosagem de HDL, glicemia de jejum e TG: HDL dosado utilizando-se um método homogêneo sem etapas de centrifugação. Complexos antígeno-anticorpos foram formados com as lipoproteínas LDL, VLDL e quilomícrons, de maneira que somente o HDL foi determinado seletivamente através de uma reação enzimática; Metodologia utilizada para a dosagem da glicemia foi oxidase, com o reagente da $A B B O T T$, onde a amostra foi coletada com técnicas de punção venosa padrão em tubos com gel separador; TG avaliados pela metodologia glicerol fosfato oxidase. Nesse tipo de reação, o mesmo é hidrolisado enzimaticamente pela lipase formando ácidos graxos livres e glicerol (ALBERT et al., 2009).

Para classificação da SM foram utilizados os critérios do IDF. Consideraram-se valores alterados: glicemia $\geq 100 \mathrm{mg} / \mathrm{dL}$, $\mathrm{HDL}<40 \mathrm{mg} / \mathrm{dL}$ em homens ou $<50 \mathrm{mg} / \mathrm{dL}$ em mulheres, TG $\geq 150 \mathrm{mg} / \mathrm{dL}, \mathrm{CC} \geq 90 \mathrm{~cm}$ no sexo masculino ou $\geq 80 \mathrm{~cm}$ no sexo feminino, PAS $\geq 130 \mathrm{mmHg}$ e PAD $\geq 85 \mathrm{mmHg}$ (INTERNATIONAL DIABETES FEDERATION, 2006). Na análise estatística os dados foram compilados no programa Microsoft Office Excel versão 2016 e foram obtidas variáveis qualitativas nominais e variáveis quantitativas. Para as variáveis qualitativas a análise descritiva procedeu com a categorização dos dados e obtenção das respectivas frequências e percentuais, e para as variáveis quantitativas foram calculadas as medianas com os respectivos intervalos de interquartis.

Para verificar a variável qualitativa SM foi utilizado o teste qui-quadrado de Pearson. O cruzamento entre as variáveis quantitativas (idade, peso, $C C$ e IMC) foi verificado inicialmente a normalidade da distribuição destas variáveis através do teste de Shapiro-Wilk e como o pressuposto não foi atendido, realizou-se os testes não paramétricos, como o de Kruskal-Wallis para comparações múltiplas e o teste de Dunn para determinar quais desses grupos são diferentes. Em todos os testes de hipótese realizados a conclusão foi obtida através da interpretação do p-valor. Adotando um nível de significância de $5 \%$ e sempre que 0 pvalor calculado for menor que 0,05 há associação entre as variáveis analisadas. O software utilizado foi o $R$, versão 3.6.1.

\section{RESULTADOS}

Foram avaliados 570 pacientes, sendo que destes 43 atenderam aos critérios de exclusão, totalizando uma amostra final de 527. A idade mínima dos pacientes analisados foi de 14 anos e máxima de 89 anos, apresentando uma idade média de $39,7( \pm 13,5)$ anos. A maioria dos pacientes era do sexo feminino $(n=356 ; 67,6 \%)$ enquanto 171 eram do sexo masculino. Com relação à presença da síndrome metabólica 108 pacientes eram portadores dessa afecção e 419 não apresentavam manifestações compatíveis (Tabela 1).

Tabela 1 - Frequência absoluta e relativa das variáveis categóricas dos pacientes analisados.

\begin{tabular}{ccc}
\hline Variável/Categorias & Frequência & Percentual \\
\hline Sexo & \\
\hline
\end{tabular}




\begin{tabular}{ccc}
\hline Feminino & 356 & 67,6 \\
Masculino & 171 & 32,4 \\
\hline Síndrome Metabólica & & \\
\hline Sim & 108 & 20,5 \\
Não & 419 & 79,5 \\
\hline
\end{tabular}

Fonte: NORONHA VFCM, et al., 2020.

A maioria dos pacientes não apresentou nenhum grau de infiltração gordurosa hepática ( $n=370 ; 70,9 \%)$ e apenas $29,1 \%$ foram classificados com algum grau de esteatose hepática (1, 2 ou 3) (Gráfico 1).

Gráfico 1 - Frequência relativa dos pacientes de acordo com os graus de esteatose hepática não alcoólica diagnosticada pela ultrassonografia abdominal.

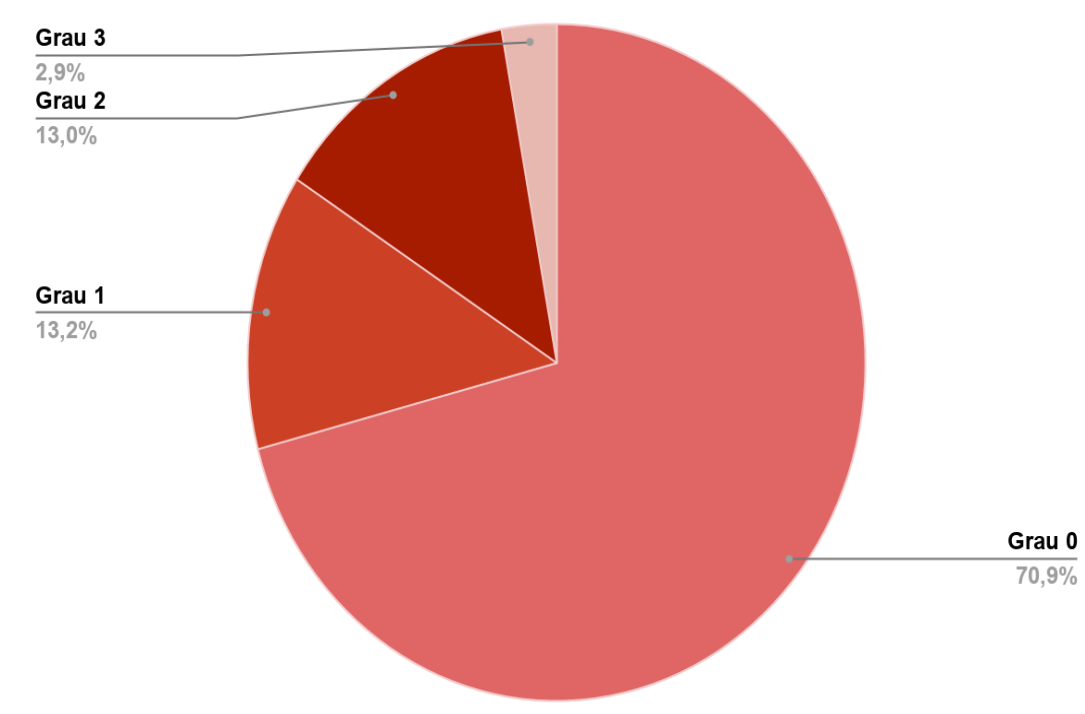

Fonte: NORONHA VFCM, et al., 2020.

A mediana do IMC foi de $22,90 \mathrm{~kg} / \mathrm{m}^{2}$ e a CC apresentou uma mediana de $78 \mathrm{~cm}$ (Tabela 2).

Tabela 2 - Mediana das variáveis numéricas dos pacientes analisados.

\begin{tabular}{lccc}
\hline \multirow{2}{*}{ Variável } & \multirow{2}{*}{ Mediana } & \multicolumn{2}{c}{ Intervalo de interquartis } \\
\cline { 3 - 4 } & & $\mathbf{2 5}$ & $\mathbf{7 5}$ \\
\hline Peso $(\mathrm{kg})$ & 60,80 & 52,28 & 72,03 \\
Altura $(\mathrm{m})$ & 1,61 & 1,56 & 1,68 \\
IMC $\left(\mathrm{kg} / \mathrm{m}^{2}\right)$ & 22,90 & 20,19 & 27,30 \\
CC $(\mathrm{cm})$ & 78,00 & 72,00 & 90,00 \\
\hline
\end{tabular}

Legenda: IMC: índice de massa corpórea; CC: circunferência da cintura. Fonte: NORONHA VFCM, et al., 2020.

Houve associação estatisticamente significativa quanto a SM e o grau da esteatose hepática não alcoólica (Tabela 3). Logo, podemos observar que pessoas sem SM tem uma tendência menor de apresentar infiltração gordurosa hepática.

Tabela 3 - Frequência absoluta e relativa da variável categórica em relação aos graus de esteatose hepática não alcoólica diagnosticada pela ultrassonografia abdominal. 


\begin{tabular}{|c|c|c|c|c|c|c|c|c|c|}
\hline & \multicolumn{2}{|c|}{0} & \multicolumn{2}{|c|}{1} & \multicolumn{2}{|c|}{2} & \multicolumn{2}{|c|}{3} & \multirow{2}{*}{ p-valor } \\
\hline & $\mathbf{N}$ & $\%$ & $\mathbf{N}$ & $\%$ & $\mathrm{~N}$ & $\%$ & $\mathrm{~N}$ & $\%$ & \\
\hline Síndrome Metabólica & & & & & & & & & 0,0001 \\
\hline Sim & 9 & 2,4 & 29 & 42,0 & 55 & 80,9 & 14 & 93,3 & \\
\hline Não & 361 & 97,6 & 40 & 58,0 & 13 & 19,1 & 1 & 6,7 & \\
\hline
\end{tabular}

Legenda: $p=$ significância estatística. Fonte: NORONHA VFCM, et al., 2020.

Observou-se diferença estatisticamente significativa do peso, circunferência da cintura e IMC em relação aos graus de esteatose hepática não alcoólica (Tabela 4). Logo a mediana do IMC dos pacientes que foram classificados como grau 0 de infiltração gordurosa foi menor que dos grau 1, 2 e 3 .

Tabela 4 - Variáveis numéricas em relação aos graus de esteatose hepática não alcoólica não alcoólica diagnosticada pela ultrassonografia abdominal.

\begin{tabular}{lccccc}
\hline Variável & Grau 0 & Grau 1 & Grau 2 & Grau 3 & p-valor \\
\hline Peso $(\mathrm{kg})$ & 55,7 & 72,4 & 79,8 & 91,5 & 0,0001 \\
$\mathrm{IMC}\left(\mathrm{kg} / \mathrm{m}^{2}\right)$ & 21,5 & 28,5 & 30,5 & 33,6 & 0,0001 \\
CC $(\mathrm{cm})$ & 75,0 & 95,4 & 101,3 & 109,0 & 0,0001 \\
\hline
\end{tabular}

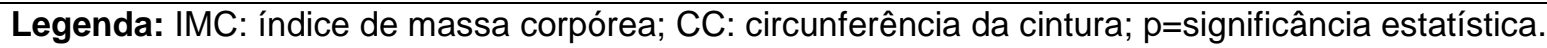

Fonte: NORONHA VFCM, et al., 2020.

\section{DISCUSSÃO}

A DHGNA é uma das doenças hepáticas mais comuns, sendo mais prevalente no mundo ocidental, afetando cerca de $24 \%$ da população mundial e até $80 \%$ das pessoas obesas (REDDY YK, et al., 2019; KIRKEGAARD-KLITBO DM, et al., 2020; NARANKIEWICZ D, et al., 2020). Para Federico A, et al. (2020), a DHGNA será a hepatopatia mais importante, sendo a primeira causa de $\mathrm{CHC}$ e transplante de fígado. Essa condição hepática é a principal causa de morbidade no mundo ocidental, superando infecções virais pelo vírus das hepatites $B$ e $C$ por conta das terapias virais existentes (YE Q, et al., 2020).

O presente estudo encontrou uma associação estatisticamente significativa entre os diferentes graus da esteatose hepática não alcoólica e SM. Trovato FM, et al. (2019) afirmaram que a DHGNA tem a SM e a RI como fatores essenciais para o acúmulo de TG intra-hepático e inflamação, conforme evidenciado também por Tan HL, et al. (2020) ao descrever a patogênese da doença. Jennison E, et al. (2019) trouxeram a DHGNA como um fator de risco independente para DM2 e doença cardiovascular e Sinha S, et al. (2020) demonstraram que a prevalência geral de SM entre pacientes com DHGNA foi bastante elevado $(64,2 \%)$ e que a SM foi significativamente mais prevalente entre os DHGNA obesos $(91,6 \%)$ do que os DHGNA magros (2,7\%). A infiltração de gordura hepática e SM compartilham uma fisiopatologia em comum, tendo a RI como papel de destaque.

Nesta pesquisa, o IMC apresentou-se estatisticamente significativo em relação aos graus de esteatose hepática. Da Silva Barbosa F e Almeida MEF (2019), identificaram que a DHGNA teve uma maior prevalência em homens, nos indivíduos com Índice de Massa Corporal $\geq 40 \mathrm{~kg} / \mathrm{m}^{2}$, dislipidêmicos (elevados teores de colesterol total, LDL, e reduzido de HDL) e com aumento da CC. Na pesquisa de Lira Neto et al. (2018) o IMC mostrou-se estatisticamente significativo em relação aos graus de DHGNA e concluíram que a obesidade potencializa condições cardiometabólicas em geral, podendo isto ser explicado pelo fato de que a obesidade cursa com alterações inflamatórias que torna o tecido adiposo resistente à insulina. A obesidade é um fator de risco para o desenvolvimento e progressão da DHGNA.

A presente pesquisa encontrou uma relação de relevância estatística entre aumento da CC e graus da esteatose hepática não alcoólica. Da Silva Barbosa F e Almeida MEF (2019) mostraram que a CC é considerada um preditor de gravidade da DHGNA. Clemente APG, et al. (2016) constataram que em adolescentes quanto maior a CC maior a prevalência de DHGNA. A medida da circunferência da cintura pode ser utilizada com segurança para estratificar o risco da infiltração gordurosa hepática, sendo uma ferramenta de triagem de baixo custo na população. 
Apesar desta pesquisa não avaliar individualmente as variáveis HAS, DM2, TG e HDL, por meio do diagnóstico da SM pelo IDF elas puderam ser analisadas e constatou-se que se associaram com DHGNA e os graus mais avançados de esteatose hepática não alcoólica. Jenisson E, et al. (2019) afirmaram que dois terços das pessoas com obesidade e DM2 e metade dos pacientes com hiperlipidemia e HAS têm gordura identificada na ultrassonografia do fígado. Também, mostraram que a presença de múltiplas características da SM está associada à doença hepática relacionada com DHGNA mais grave e maior probabilidade de progressão para EHNA e fibrose hepática.

\section{CONCLUSÃO}

Constatou-se uma associação estatisticamente significativa entre DHGNA e SM na população estudada. A SM evidenciou ser um importante fator para maior gravidade da DHGNA diagnosticada pela USG. Em relação aos dados antropométricos (peso, IMC e CC), quanto maiores as suas medianas, maiores os graus de esteatose hepática não alcoólica. De acordo com o IMC, indivíduos com sobrepeso tiveram maior risco de ter esteatose hepática grau 1 e os obesos relacionaram-se mais aos graus 2 e 3. Conforme a CC, indivíduos com parâmetros adequados classificaram-se com ecogenicidade hepática normal na USG e os com a CC acima do recomendado revelaram maior risco de graus de esteatose no fígado. Logo, métodos não invasivos e de baixo custo, como as medidas antropométricos e a classificação de SM, mostraram ser eficientes ferramentas de triagem e podem permitir o direcionamento de pacientes mais vulneráveis à progressão e gravidade da DHGNA.

\section{REFERÊNCIAS}

1. BATTAGLIA S, et al. Gender, BMI and fasting hyperglycaemia influence Monocyte to-HDL ratio (MHR) index in metabolic subjects. PloS one, 2020; 15 (4).

2. CLEMENTE APG, et al. Circunferência da cintura como marcador para triagem de doença hepática gordurosa não alcoólica em adolescentes obesos. Revista Paulista de Pediatria, 2016; 34 (1): 47-55.

3. DA SILVA BARBOSA F, ALMEIDA MEF. Doença Hepática Gordurosa Não Alcoólica: um problema global de caráter reversível. Journal of Health \& Biological Sciences, 2019; 7 (3): 305-311.

4. DIETRICH P, HELLERBRAND C. Non-alcoholic fatty liver disease, obesity and the metabolic syndrome. Best Pract Res Clin Gastroenterol, 2014; 28 (4):637-653.

5. FEDERICO A, et al. The Bisphenol A Induced Oxidative Stress in Non-Alcoholic Fatty Liver Disease Male Patients: A Clinical Strategy to Antagonize the Progression of the Disease. International Journal of Environmental Research and Public Health, 2020; 17 (10): 3369.

6. HUANG T, et al. Non-alcoholic fatty liver disease (NAFLD): a review of epidemiology, risk factors, diagnosis and management. Internal medicine journal, 2019.

7. INTERNATIONAL DIABETES FEDERATION. The IDF consensus worldwide definition of the metabolic syndrome. IDF Communications, 2006.

8. JENNISON E, et al. Diagnosis and management of non-alcoholic fatty liver disease. Postgraduate medical journal, 2019; 95 (1124): 314-322

9. KIRKEGAARD-KLITBO DM, et al. Prevalence and Risk Factors of Moderate to Severe Hepatic Steatosis in HIV Infection: The Copenhagen Co-Morbidity Liver Study. The Journal Of Infectious Diseases, 2020.

10. LIRA NETO JCG et al. Prevalência da síndrome metabólica e de seus componentes em pessoas com diabetes mellitus tipo 2. Universidade Federal do Piauí, Departamento de Enfermagem, 2018; 27 (3).

11. LOHMAN TG, et al. Anthropometric standardization reference manual. Champaign: Human kinetics books, 1988.

12. MINCIS M, MINCIS R. Álcool e fígado. Gastroenterologia Endoscopia Digestiva (GED) 2011; 30(4): 152-62.

13. NARANKIEWICZ D, et al. Utility of Liver Function Tests and Fatty Liver Index to Categorize Metabolic Phenotypes in a Mediterranean Population. International Journal of Environmental Research and Public Health, 2020; 17 (10): 3518.

14. PAPATHEODORIDI M, CHOLONGITAS E. Diagnosis of non-alcoholic fatty liver disease (NAFLD): current concepts. Current pharmaceutical design, 2018; 24 (38): 4574-4586.

15. REDDY YK, et al. Natural History of Non-Alcoholic Fatty Liver Disease: A Study With Paired Liver Biopsies. Journal of Clinical and Experimental Hepatology, 2019.

16. ROCHLANI Y, et al. Metabolic syndrome: pathophysiology, management, and modulation by natural compounds. Therapeutic advances in cardiovascular disease, 2017; 11 (8): 215-225.

17. SAADEH SY, et al. The Utility of Radiological Imaging in Nonalcoholic Fatty Liver Disease. Gastroenterology, 2002; $123(3): 745-750$.

18. SOCIEDADE BRASILEIRA DE CARDIOLOGIA et al. $7^{a \underline{a}}$ Diretriz Brasileira de Hipertensão Arterial. Arquivos Brasileiros de Cardiologia, 2018; 107 (3). 
19. SINHA N, et al. Metabolic syndrome is not uncommon among lean non-alcoholic fatty liver disease patients as compared with those with obesity. Indian J Gastroenterol, 2020; 39: 75-83.

20. SWEET PH, et al. Nonalcoholic fatty liver disease. Primary Care: Clinics in Office Practice, $2017 ; 44$ (4): $599-607$.

21. TAN HL, et al. HLA-DQB1* 06 reduces fibrosis score in patients with non-alcoholic fatty liver disease. Hepatology Research: the Official Journal of the Japan Society of Hepatology, 2020.

22. TREFTS E, et al. The liver. Current Biology, 2017; 27 (21).

23. TROVATO FM, et al. Nonalcoholic fatty liver disease (NAFLD) prevention: role of Mediterranean diet and physical activity. Hepatobiliary surgery and nutrition, 2019, 8 (2): 167-169.

24. WANG L, et al. Low Serum ZAG Levels Correlate With Determinants of the Metabolic Syndrome in Chinese Subjects. Frontiers in Endocrinology, 2020; 11: 154.

25. WHO. WORLD HEALTH ORGANIZATION. Physical status: the use and interpretation of anthropometry.Geneva, 1995.

26. YE Q, et al. Global prevalence, incidence, and outcomes of non-obese or lean non-alcoholic fatty liver disease: a systematic review and meta-analysis. The Lancet Gastroenterology \& Hepatology, 2020.

27. YKI-JÄRVINEN H. Non-alcoholic fatty liver disease as a cause and a consequence of metabolic syndrome. Lancet Diabetes Endocrinol, 2014; 2 (11):901-910. 